\title{
Stochastic background of relic gravitons in a bouncing quantum cosmological model
}

\author{
Dennis Bessada ${ }^{1,3}$, Nelson Pinto-Neto ${ }^{2 \ddagger}$, Beatriz B. Siffert ${ }^{2,4 \dagger}$ and Oswaldo D. Miranda ${ }^{3 \S}$ \\ ${ }^{1}$ UNIFESP - Universidade Federal de São Paulo - Laboratório de Física Teórica e Computação Científica, \\ Rua São Nicolau, 210, 09913-030, Diadema, SP, Brazil \\ ${ }^{2}$ ICRA - CBPF - Centro Brasileiro de Pesquisas Físicas - Rua \\ Dr. Xavier Sigaud, 150, Urca, 22290-180, Rio de Janeiro, Brazil \\ ${ }^{3}$ INPE - Instituto Nacional de Pesquisas Espaciais - Av. dos Astronautas, \\ 1758, 1222\%-010, São José dos Campos, SP, Brazil \\ ${ }^{4}$ Instituto de Física - Universidade Federal do Rio de Janeiro - \\ Av. Athos da Silveira Ramos 149, 21941-972, Rio de Janeiro, Brazil
}

\begin{abstract}
The spectrum and amplitude of the stochastic background of relic gravitons produced in a bouncing universe is calculated. The matter content of the model consists of dust and radiation fluids, and the bounce occurs due to quantum cosmological effects when the universe approaches the classical singularity in the contracting phase. The resulting amplitude is very small and it cannot be observed by any present and near future gravitational wave detector. Hence, as in the ekpyrotic model, any observation of these relic gravitons will rule out this type of quantum cosmological bouncing model.
\end{abstract}

PACS numbers: $98.80 . \mathrm{Cq}$

\section{INTRODUCTION}

Bouncing cosmological models [1] are being widely investigated because, besides solving the singularity problem in cosmology by construction, they can also solve the horizon and flatness puzzles, and lead to an almost scale invariant spectrum of scalar perturbations if the contracting phase is dominated by dust at large scales [2].

One of the calculations that have been done was the evaluation of the spectral index of long wavelength tensor perturbations, $n_{T}$, and in most of bouncing models they were found to be also scale invariant. With respect to their amplitudes, specific models must be worked out. For instance, in the cyclic ekpyrotic scenario, they were evaluated and it was shown that the amplitudes are too small to be detected by present gravitational wave detectors [3].

In this paper we will calculate the spectrum and amplitude of relic gravitons in a different bouncing cosmological model. It consists of a Friedmann-LemaîtreRobertson-Walker (FLRW) universe filled by dust and radiation, which is contracting classically. As it approaches the singularity, quantum cosmological effects on the background, here described through the WheelerDeWitt equation interpreted along the lines of the Bohmde Broglie quantum theory, avoids the singularity and ejects the universe to the expanding phase we are now experiencing. Note that our bouncing model is very conservative: there is nothing else than dust and radiation, we are working with a $3+1$ dimensional space-time, and we are performing a canonical quantization of the second order perturbed (with respect to the FLRW background) Einstein-Hilbert action of general relativity, interpreted along the lines of a quantum theory appropriate to quantum cosmology, namely, one which does not need any external agent to the quantum system to give a meaning to the quantum calculations.
Evolution of quantum perturbations (scalar, vector and tensor) on these quantum backgrounds can be described by simple equations, as it was demonstrated in Refs. [4, 5]. With these equations, we were able to calculate the spectrum (analytically and numerically) and amplitude (numerically) of this stochastic background of relic gravitons. Although, the spectrum and amplitude differ considerably from the cyclic ekpyrotic scenario, the main conclusion remains the same, namely, that the amplitudes are too small to be detected by any present and near future gravitational wave detectors.

The paper is divided as follows: in the next section we review the main aspects of the quantum cosmological bouncing model on which the relic gravitons evolves, and we obtain the dynamical equations that the tensor perturbations which describe these relic gravitons must obey. In section III we derive the expression for the critical fraction of the relic gravitons energy density from the tensor perturbations described in section II. In section IV we calculate the spectrum and amplitude of this energy density and the graviton strain either analytically (for the spectrum) and numerically. We end up with the conclusions.

\section{THE STOCHASTIC BACKGROUND OF RELIC GRAVITONS IN A BOUNCING QUANTUM COSMOLOGICAL MODEL}

We start reviewing the key ideas concerning tensor perturbations in a perfect fluid quantum cosmological model in the Bohm-de Broglie interpretation as put forward in Refs. [4, [6]. 


\section{A. Hamiltonian formalism for tensor perturbations}

We start with an Einstein-Hilbert action coupled to a perfect fluid described by the Schutz formalism [7]:

$$
S=S_{\mathrm{GR}}+S_{\text {fluid }}=-\frac{1}{6 \ell_{\mathrm{P} 1}^{2}} \int \sqrt{-g} R \mathrm{~d}^{4} x+\int \sqrt{-g} P \mathrm{~d}^{4} x,
$$

where $\ell_{\mathrm{P} 1}=\left(8 \pi G_{\mathrm{N}} / 3\right)^{1 / 2}$ is the Planck length in natural units $(\hbar=c=1), P$ is the perfect fluid pressure whose density $\rho$ is given by the equation of state $P=\omega \rho$, with $\omega=$ const.

Writing down the action of a fluid as proportional to the pressure has also been proposed by other authors [8]. All these approaches are generally covariant, but they yield a preferred time direction, the one connected with the surfaces of constant potential of the velocity field of the fluid.

Such very simple fluids can also be completely characterized by the k-essence lagrangian $\left(\partial_{\mu} \phi \partial^{\mu} \phi\right)^{(w+1) /(2 w)}$, where $\phi$ is a scalar field and $w$ is a constant. This scalar field has equation of state $p=w \rho$. It has no potential and a non-trivial kinetic term. The matter Hamiltonian which we will exhibit below can be easily obtained from this Lagrangian after one performs a simple canonical transformation.
This fluid description is suitable to describe the primordial universe, when radiation dominates and all particles become relativistic, if we make the choice $w=1 / 3$. In what folows, we will directly quantize this perfect fluid Lagrangian. This can be justified in physical grounds because it was implementes while studying superfluids [9], and the quantum model based on this procedure turned out to be quite accurate in describing many properties of such physical systems.

The metric $\boldsymbol{g}$ in Eq. (1) is decomposed into a background Friedman-Lemaître-Robertson-Walker (FLRW) metric and into a first-order tensor perturbation $w_{i j}$, and is given in the Arnowitt-Deser-Misner (ADM) formalism by

$$
\mathrm{d} s^{2}=N^{2}(\tau) \mathrm{d} \tau^{2}-a_{\text {phys }}^{2}(\tau)\left(\gamma_{i j}+w_{i j}\right) \mathrm{d} x^{i} \mathrm{~d} x^{j} .
$$

The background metric $\gamma_{i j}$ is related to the spacelike hypersurfaces with constant curvature $\mathcal{K}(\mathcal{K}=0, \pm 1$ for flat, open and closed space respectively), and lowers and raises the indices of the tensor perturbation $w_{i j}$, which is transverse and traceless (i.e. , $w^{i j}{ }_{\mid j}=0$ and $w_{i}^{i}=0$, where the bar indicates a covariant derivative with respect to $\gamma) . N(\tau)$ is the lapse function and defines the gauge, fixed once and for all.

The second-order Hamiltonian for the gravitational model described by the action (1) and metric (2) can be written as (see Ref. [6] for further details)

$$
\begin{aligned}
H \equiv & N H_{0} \\
= & N\left\{-\frac{P_{a}^{2}}{4 a}-\mathcal{K} a+\frac{P_{T}}{a^{3 \omega}}\left(1+\frac{\omega}{4} \int \mathrm{d}^{3} x \gamma^{1 / 2} w_{i j} w^{i j}\right)+\frac{5 P_{a}^{2}}{48 a} \int \mathrm{d}^{3} x \gamma^{1 / 2} w_{i j} w^{i j}\right. \\
& \left.+\int \mathrm{d}^{3} x\left[\frac{6 \Pi_{i j} \Pi^{i j}}{a^{3} \gamma^{1 / 2}}+2 \frac{P_{a} w_{i j} \Pi^{i j}}{a^{2}}+\gamma^{1 / 2} a\left(\frac{w^{i j \mid k} w_{i j \mid k}}{24}+\frac{\mathcal{K}}{6} w_{i j} w^{i j}\right)\right]\right\},
\end{aligned}
$$

where the quantities $P_{a}, \Pi^{i j}, P_{T}$ are the momenta canonically conjugate to the scale factor, the tensor perturbations, and to the fluid degree of freedom, respectively. These quantities have been redefined in order to be dimensionless. For instance, the physical scale factor $a_{\text {phys }}$ can be obtained from the dimensionless $a$ present in (3) through $a_{\mathrm{phys}}=\ell_{\mathrm{Pl}} a / \sqrt{V}$, where $V$ is the comoving volume of the background spacelike hypersurfaces. This Hamiltonian, which is zero due to the constraint $H_{0} \approx 0$, yields the correct Einstein equations both at zeroth and first order in the perturbations, as can be checked explicitly. In order to obtain its expression, no assumption has been made about the background dynamics, just Legendre and canonical transformations have been performed.

The fact that the momentum $P_{T}$ appears linearly in the Hamiltonian suggests to consider its canonical position $T$ as a time variable. Indeed, from the canonical transformation used to arrive at this expression [8], one has that $\phi=T,\left(L_{m}=\left(\partial_{\mu} \phi \partial^{\mu} \phi\right)^{(w+1) /(2 w)}\right)$ that is, it is just the potential of the fluid velocity, which characterizes a prefered foliation of spacetime. This time variable always increases, as it can be checked in the contracting and expanding classical solutions, and in the Bohmian bounce solution we present below.

In the quantum regime, this Hamiltonian can be substantially simplified through the implementation of the quantum canonical transformation generated by

$$
U=\exp \left(i G_{\mathrm{q}}\right) \equiv \exp \left(\frac{i}{12} \hat{\beta}_{a} \hat{Q}\right)
$$

where $\hat{\beta}_{a} \equiv \frac{1}{2}\left(\hat{P}_{a} \hat{a}+\hat{a} \hat{P}_{a}\right)$ and $\hat{Q} \equiv \int \mathrm{d}^{3} x \gamma^{1 / 2} \hat{w}_{i j} \hat{w}^{i j}$ are the self-adjoint operators associated with the corresponding classical variables, yielding, for a particular factor ordering of (3) (see Section 3 in Ref. [6] for further 
details), the following quantum Hamiltonian

$$
\begin{aligned}
\hat{H}_{0} & =\left[-\frac{1}{4 \hat{a}} \hat{P}_{a}^{2}-\mathcal{K} \hat{a}+\frac{\hat{P}_{T}}{\hat{a}^{3 \omega}}+\int \mathrm{d}^{3} x\left(6 \frac{\hat{\Pi}^{i j} \hat{\Pi}_{i j}}{\gamma^{1 / 2} a^{3}}\right.\right. \\
& \left.\left.+\frac{1}{24} \gamma^{1 / 2} a \hat{w}_{i j \mid k} \hat{w}^{i j \mid k}+\frac{1}{12} \gamma^{1 / 2} \mathcal{K} \hat{w}_{i j} \hat{w}^{i j} a\right)\right] .
\end{aligned}
$$

Performing an inverse Legendre transform on the second-order piece of the Hamiltonian (5), and restoring the constant $\ell_{\mathrm{P} 1}$, we get the following Lagrangian density

$$
\mathcal{L}^{(2)}=\frac{1}{24 \ell_{\mathrm{P} 1}^{2}} \sqrt{-{ }^{(0)} g}\left[{ }^{(0)} g^{\alpha \beta} w^{i}{ }_{j \mid \alpha} w_{i}{ }^{j \mid \beta}-2 \mathcal{K} w^{i}{ }_{j} w^{j}{ }_{i}\right](, 6)
$$

where ${ }^{(0)} g_{\alpha \beta}$ is the background piece of the full metric (2). Note that Lagrangian (6) coincides with the one derived in [10] for classical backgrounds.

\section{B. Quantum evolution of the background and perturbation variables}

The quantization procedure of the background and tensor perturbations can be implemented by imposing $\hat{H}_{0} \Psi\left(a, w_{i j}\right)=0$. The Wheeler-DeWitt equation in this case reads

$$
\begin{aligned}
i \frac{\partial \Psi}{\partial T} & =\hat{H}_{\mathrm{red}} \Psi \\
& \equiv\left\{\frac{a^{3 \omega-1}}{4} \frac{\partial^{2}}{\partial a^{2}}-\mathcal{K} a^{3 \omega+1}+\int \mathrm{d}^{3} x\left[-6 \frac{a^{3(\omega-1)}}{\gamma^{1 / 2}} \frac{\delta^{2}}{\delta w_{i j} \delta w^{i j}}+a^{3 \omega+1}\left(\gamma^{1 / 2} \frac{w_{i j \mid k} w^{i j \mid k}}{24}+\mathcal{K} \frac{w_{i j} w^{i j}}{12}\right)\right]\right\}
\end{aligned}
$$

where we have chosen $T$ as the time variable, which is equivalent to impose the time gauge $N=a^{3 \omega}$. Next, using the Bohm-de Broglie interpretation of quantum mechanics [11], making the separation ansatz for the wave functional $\Psi\left[a, w_{i j}, T\right]=\varphi(a, T) \psi\left[a, w_{i j}, T\right]$, and following the reasoning of Ref. [12], we can show that Eq. (7) can be split into two, namely

$$
i \frac{\partial \varphi}{\partial T}=\frac{a^{3 \omega-1}}{4} \frac{\partial^{2} \varphi}{\partial a^{2}}-\mathcal{K} a^{3 \omega+1} \varphi
$$

and

$$
\begin{aligned}
i \frac{\partial \psi}{\partial T} & =\int \mathrm{d}^{3} x\left[-6 \frac{a^{3(\omega-1)}}{\gamma^{1 / 2}} \frac{\delta^{2}}{\delta w_{i j} \delta w^{i j}}\right. \\
& \left.+a^{3 \omega+1}\left(\gamma^{1 / 2} \frac{w_{i j \mid k} w^{i j \mid k}}{24}+\mathcal{K} \frac{w_{i j} w^{i j}}{12}\right)\right] \psi
\end{aligned}
$$

Using the Bohm-de Broglie interpretation, Eq. (8) can now be solved as in Refs. [13 15], yielding a Bohmian quantum trajectory $a(T)$, which in turn can be used to simplify Eq. (9) 6]. Indeed, as one can understand $a(T)$ as a prescribed function of time, which implies that $\psi\left(a, w_{i j}, T\right)=\psi\left(a(T), w_{i j}, T\right)=\psi^{\prime}\left(w_{i j}, T\right)$, one can perform the time dependent unitary transformation

$$
U=\exp \left\{i\left[\int d^{3} x \gamma^{1 / 2} \frac{a^{\prime} w_{i j} w^{i j}}{2 a}\right]\right\} \exp \left\{i\left[\int d^{3} x\left(\frac{w_{i j} \Pi^{i j}+\Pi^{i j} w_{i j}}{2}\right) \ln \left(\frac{\sqrt{12}}{a}\right)\right]\right\}
$$

yielding the following simple form for the Schrödinger equation for the perturbations:

$$
i \frac{\partial \varphi(w, \eta)}{\partial \eta}=\int d^{3} x\left\{-\frac{1}{2 \gamma^{1 / 2}} \frac{\delta^{2}}{\delta w^{2}}+\gamma^{1 / 2}\left[\frac{1}{2} w_{k} w^{k}-\frac{a^{\prime \prime}}{2 a} w^{2}\right]\right\} \varphi(w, \eta) .
$$

\footnotetext{
${ }^{1}$ We are assuming that there is a disentangled zeroth order term in the total wave function. It is a reasonable physical assumption as long as the semi-classical theory of cosmological perturbations, where the background behaves classically and perturbations are quantum, seems to describe quite well our real universe. This would be impossible if the wave function were completely entan-
}

gled. Note that there is no definite theory of initial conditions for the wave function of the universe, hence one must rely in such post-factum assumptions, one, namely, that our universe has a classical limit. 
A transformation to conformal time $\eta, a^{3 \omega-1} \mathrm{~d} T=\mathrm{d} \eta$, was also done, and a prime ' denotes the derivative with respect to $\eta$ (see again Ref. [6] for details).

This Schrödinger equation is identical to the one used in semi-classical gravity for linear tensor perturbations, but here it was obtained without ever using the background Einstein's equations. Hence it can be used when the background is also quantized. It is important to stress that the function $a(\eta)$ which is present in Eq. (11) is no longer the classical solution for the scale factor, but the quantum Bohmian solution. This fact leads to some different consequences with respect to the usual semi-classical approach, as we will see.

In the Heisenberg representation, the equations for the operator $\hat{w}_{i j}$ read

$$
\hat{w}_{i j}^{\prime \prime}+2 \frac{a^{\prime}}{a} \hat{w}_{i j}^{\prime}-\hat{w}_{i j \mid k}^{\mid k}+2 \mathcal{K} \hat{w}_{i j}=0,
$$

which corresponds to the usual equation for quantum tensor perturbations in classical backgrounds [10].

We can proceed with the usual analysis, but now taking the quantum Bohmian solution $a(\eta)$ coming from Eq. (8) as the new pump field. In order to obtain these background quantum solutions, we follow the procedure discussed in Ref. [4] which, for flat spatial sections $(\mathcal{K}=0)$, yields the following guidance relation

$$
\frac{d a}{d T}=-\frac{a^{(3 \omega-1)}}{2} \frac{\partial S}{\partial a}
$$

in accordance with the classical relations $d a / d T=\{a, H\}=-\frac{1}{2} a^{(3 \omega-1)} P_{a}$ and $P_{a}=\partial S / \partial a$. For the wave function

$$
\Psi(a, T)=\left[\frac{8 T_{b}}{\pi\left(T^{2}+T_{b}^{2}\right)}\right]^{1 / 4} \exp \left[\frac{-4 T_{b} a^{3(1-\omega)}}{9\left(T^{2}+T_{b}^{2}\right)(1-\omega)^{2}}\right] \exp \left\{-i\left[\frac{4 T a^{3(1-\omega)}}{9\left(T^{2}+T_{b}^{2}\right)(1-\omega)^{2}}+\frac{1}{2} \arctan \left(\frac{T_{b}}{T}\right)-\frac{\pi}{4}\right]\right\}
$$

which comes from the initial normalized gaussian at $T=0$

$$
\Psi^{(\text {init })}(\chi)=\left(\frac{8}{T_{b} \pi}\right)^{1 / 4} \exp \left(-\frac{\chi^{2}}{T_{b}}\right)
$$

where $T_{b}$ is an arbitrary constant, the Bohmian quantum trajectory for the scale factor is given by

$$
a(T)=a_{b}\left[1+\left(\frac{T}{T_{b}}\right)^{2}\right]^{\frac{1}{3(1-\omega)}}
$$

where $a_{b}$ is the minimum value for the scale factor at the bounce $T=0$. Note that this solution has no singularities and tends to the classical solution when $T \rightarrow \pm \infty$. The quantity $T_{b}$, which is the width of the initial gaussian (15), yields the curvature scale at the bounce, $L_{\text {bounce }} \equiv T_{b} a_{b}^{3 w}$. There is nothing, at this stage of quantum cosmology, which may constrain its value: we have not yet a theory of initial conditions. It is a free parameter. However, physical considerations allow us to say that it must be greater than the Planck length but smaller than the curvature scale when nucleosynthesis takes place, in order to not spoil its results.

The tensor perturbations are quantum-mechanical operators, hence it is convenient to expand them into Fourier modes and subject them to quantization rules:

$$
\begin{aligned}
\hat{w}_{i j}(x) & =\sqrt{6} \ell_{\mathrm{P} 1} \sum_{\lambda=+, \times} \int \frac{d^{3} k}{(2 \pi)^{3 / 2}} \varepsilon_{i j}^{(\lambda)}\left[w_{k}^{(\lambda)}(\eta) e^{-i \mathbf{k} \cdot \mathbf{x}} \hat{a}_{\mathbf{k}}^{(\lambda)}\right. \\
& \left.+w_{k}^{*(\lambda)}(\eta) e^{i \mathbf{k} \cdot \mathbf{x}} \hat{a}_{\mathbf{k}}^{(\lambda) \dagger}\right]
\end{aligned}
$$

where $x=(\eta, \mathbf{x}), \varepsilon_{i j}^{(\lambda)}=\varepsilon_{i j}^{(\lambda)}(\hat{\mathbf{k}})$ is the polarization tensor for the two graviton polarization states + and $\times$ labeled by $\lambda$, and satisfies

$$
\varepsilon^{(\lambda) i j} \varepsilon_{i j}^{\left(\lambda^{\prime}\right)}=2 \delta_{\lambda \lambda^{\prime}} .
$$

Also, $w_{k}^{(\lambda)}(\eta)$ are mode functions, and $\hat{a}_{\mathbf{k}}^{(\lambda) \dagger}, \hat{a}_{\mathbf{k}}^{(\lambda)}$ are creation and annihilation operators, respectively. Such operators satisfy the equal-time commutation relations

$$
\begin{aligned}
& {\left[\hat{a}_{\mathbf{k}}^{(\lambda)}, \hat{a}_{\mathbf{k}^{\prime}}^{\left(\lambda^{\prime}\right) \dagger}\right]=\delta_{\lambda \lambda^{\prime}} \delta^{(3)}\left(\mathbf{k}-\mathbf{k}^{\prime}\right),} \\
& {\left[\hat{a}_{\mathbf{k}}^{(\lambda)}, \hat{a}_{\mathbf{k}^{\prime}}^{\left(\lambda^{\prime}\right)}\right]=\left[\hat{a}_{\mathbf{k}}^{(\lambda) \dagger}, \hat{a}_{\mathbf{k}^{\prime}}^{\left(\lambda^{\prime}\right) \dagger}\right]=0,}
\end{aligned}
$$

and the quantum vacuum is defined by

$$
\hat{a}_{\mathbf{k}}^{(\lambda)}|0\rangle=0 .
$$

This vacuum initial condition was chosen because the universe in the contracting phase was very big, rarefied and almost flat, where inhomogeneities are supposed to be wiped out through dissipation, see Ref. [5] on that. This is a general assumption in bouncing models.

Next, inserting the Fourier expansion into Eq. (12), we get the mode equation

$$
w_{k}^{(\lambda) \prime \prime}+2 \frac{a^{\prime}}{a} w_{k}^{(\lambda) \prime}+\left(k^{2}+2 \mathcal{K}\right) w_{k}^{(\lambda)}=0 .
$$


Introducing the canonical amplitude $v_{k}^{(\lambda)}$ as

$$
v_{k}^{(\lambda)} \equiv a w_{k}^{(\lambda)}
$$

mode equation (22) becomes

$$
v_{k}^{(\lambda)^{\prime \prime}}+\left(k^{2}+2 \mathcal{K}-\frac{a^{\prime \prime}}{a}\right) v_{k}^{(\lambda)}=0,
$$

for each graviton polarization state. In the present work, we will only be concerned with the case $\mathcal{K}=0$, i.e., a Universe with flat spatial sections.

\section{STOCHASTIC BACKGROUND OF RELIC GRAVITONS}

In the $\Lambda \mathrm{CDM}$ model, quantum fluctuations arising during inflation lead to a nearly scale-invariant spectrum of density (scalar) [16] and gravitational waves (tensor) perturbations [17]. As in the case of CMB, we also expect relic gravitons generated in this early epoch to form a background - the stochastic background of relic gravitons (SBRG) - to be hopefully detected by the high-sensitivity gravitational waves $(\mathrm{GW})$ detectors. The physical observable to be measured by such GW detectors is the critical fraction of the relic gravitons energy density $\rho_{G W}$ given by (see [18, 19] and references therein)

$$
\Omega_{G W}(\eta) \equiv \frac{\rho_{G W}(\eta)}{\rho_{c}}
$$

where $\rho_{c}=\left(H / \ell_{\mathrm{Pl}}\right)^{2}$ is the critical energy density. The energy density $\rho_{G W}$ carried by the relic gravitons is simply the 0-0 component of the stress energy-momentum tensor of the tensor perturbations, that is $\rho_{G W}=T^{0}{ }_{0}$, where

$$
T_{\alpha \beta} \equiv-\frac{2}{\sqrt{-{ }^{(0)} g}} \frac{\partial \mathcal{L}^{(2)}}{\partial^{(0)} g^{\alpha \beta}},
$$

and $\mathcal{L}^{(2)}$ is the Lagrangian (6) for the tensor perturbations (recall that Lagrangian (6) holds either in classical or quantum backgrounds). Considering only spatiallyflat models $(\mathcal{K}=0)$, the energy density of the relic gravitons can be derived by inserting (6) into (26) and taking the 0-0 component, which yields the classical expression

$$
\rho_{G W}=\frac{1}{12 \ell_{\mathrm{Pl}}^{2}}\left[\frac{1}{a^{2}} w^{i j^{\prime}} w_{i j}{ }^{\prime}-\frac{1}{2} w^{i j, \mu} w_{i j, \mu}\right] .
$$

Since tensor fluctuations generated during inflation are of quantum nature, the energy density of the relic gravitons becomes the expected value of the operator $\stackrel{T}{0}_{0}^{0}$ in the vacuum state defined in (21):

$$
\rho_{G W} \equiv\left\langle 0\left|\hat{T}_{0}^{0}\right| 0\right\rangle \text {. }
$$

Substituting the Fourier expansion (17) into (27), and the result into (28), we find, using the commutation relations (1920121), that

$$
\begin{aligned}
\rho_{G W}(\eta) & =\frac{1}{a^{2}} \sum_{\lambda} \int d \ln k \frac{k^{3}}{4 \pi^{2}}\left[\left|w_{k}^{(\lambda)^{\prime}}(\eta)\right|^{2}\right. \\
& \left.+k^{2}\left|w_{k}^{(\lambda)}(\eta)\right|^{2}\right] .
\end{aligned}
$$

In terms of the canonical field $v_{k}^{(\lambda)}$ introduced in (23), expression (29) turns into

$$
\rho_{G W}(\eta)=\int d \ln k \rho_{G W}(k, \eta),
$$

where we have introduced the energy density per mode

$$
\begin{aligned}
\rho_{G W}(k, \eta) & \equiv \frac{d \rho_{G W}(\eta)}{d \ln k} \\
& =\frac{k^{3}}{4 \pi^{2} a^{4}} \sum_{\lambda}\left\{\left|v_{k}^{(\lambda)^{\prime}}\right|^{2}-\mathcal{H}\left[v_{k}^{(\lambda)} v_{k}^{(\lambda)^{\prime} *}\right.\right. \\
& \left.\left.+v_{k}^{(\lambda) *} v_{k}^{(\lambda)^{\prime}}\right]+\left(k^{2}+\mathcal{H}^{2}\right)\left|v_{k}^{(\lambda)}\right|^{2}\right\}
\end{aligned}
$$

where $\mathcal{H}=a^{\prime} / a$ is the Hubble parameter in conformal time coordinates.

From (31) the energy density parameter (25) per mode is given by

$$
\Omega_{G W}(k, \eta)=\frac{\ell_{\mathrm{Pl}}^{2}}{2 \pi^{2} a^{2}} \frac{k^{3}}{\mathcal{H}^{2}} \sum_{\lambda}\left\{\left|v_{k}^{(\lambda)^{\prime}}\right|^{2}-\mathcal{H}\left[v_{k}^{(\lambda)} v_{k}^{(\lambda)^{\prime} *}+v_{k}^{(\lambda) *} v_{k}^{(\lambda)^{\prime}}\right]+\left(k^{2}+\mathcal{H}^{2}\right)\left|v_{k}^{(\lambda)}\right|^{2}\right\}
$$

By solving the mode equation (24) and inserting the result into (32), we promptly determine the energy density parameter per logarithmic frequency interval $\nu=$ $k /(2 \pi a)$, which is the physical quantity to be confronted with future observations.

\section{STOCHASTIC BACKGROUND OF RELIC GRAVITONS IN A DUST-RADIATION BOUNCING UNIVERSE}

\section{A. Scale Factor}

The scale factor for the radiation dominated bounce will be given by Eq. (16), with $w=1 / 3$. In this case, we 
have $d T=d \eta$ so, we find

$$
a_{\text {bounce }}(\eta)=a_{b} \sqrt{1+\left(\frac{\eta}{\eta_{b}}\right)^{2}},
$$

where $\eta_{b}$ is a free parameter that determines the duration of the bouncing phase.

The scale factor before and after the bounce is the usual classical solution for a Universe filled with radiation and dust [20],

$$
a_{\text {rad }}(\eta)=a_{e q}\left[\left(\frac{\eta}{\eta_{*}}\right)^{2} \mp 2 \frac{\eta}{\eta_{*}}\right],
$$

where the minus and plus signs refers to the epochs before and after the bounce, respectively, $a_{e q}$ is the value of the scale factor at matter-radiation equality, and

$$
\eta_{*}=2 R_{H} \frac{\sqrt{2+z_{e q}}}{1+z_{e q}}
$$

where $R_{H}=1 /\left(a_{0} H_{0}\right)$ is the co-moving Hubble radius, $a_{0}$ and $H_{0}$ are the present values of the scale factor and Hubble radius, respectively, and $z_{e q} \approx 3 \times 10^{3}$ is the value of the redshift at matter-radiation equality. As the bounce should occur much before the matter-radiation equality, then $\eta_{b}<<\eta_{*}$.

The above expressions for $a_{\text {bounce }}$ and $a_{\text {rad }}$ can be condensed into

$$
a(\eta)=a_{e q}\left[\left(\frac{\eta}{\eta_{*}}\right)^{2}+2 \frac{\eta_{b}}{\eta_{*}} \sqrt{1+\left(\frac{\eta}{\eta_{b}}\right)^{2}}\right],
$$

which promptly recovers Eqs. (33) and (34) in the limits $\eta<<\eta_{*}$ and $\eta>>\eta_{b}$, respectively, and assuming that $a_{b}=2 a_{e q} \eta_{b} / \eta_{*}$.

With this expression for the scale factor, we are ready to solve the mode equation, Eq. (24).

\section{B. Mode Equation}

For numerical purposes, we prefer to work with the normalized variables

$$
\bar{\eta} \equiv \frac{\eta}{2 R_{H}} \quad \text { and } \quad n \equiv 2 R_{H} k .
$$

In terms of these variables, the mode equation for a flat Universe becomes

$$
\frac{d^{2} v_{n}}{d \bar{\eta}^{2}}+\left[n^{2}-\frac{d^{2} a}{d \bar{\eta}^{2}} \frac{1}{a}\right] v_{n}=0,
$$

where we are treating separately each possible graviton polarization state, so we have dropped the superscript $(\lambda)$.

The scale factor given in Eq. (36) becomes

$$
a(\bar{\eta})=a_{e q}\left[\left(\frac{\bar{\eta}}{\bar{\eta}_{*}}\right)^{2}+2 \frac{\bar{\eta}_{b}}{\bar{\eta}_{*}} \sqrt{1+\left(\frac{\bar{\eta}}{\bar{\eta}_{b}}\right)^{2}}\right],
$$

where $\bar{\eta}_{b}=\eta_{b} / 2 R_{H}$ is a free parameter and

$$
\bar{\eta}_{*}=\frac{\eta_{*}}{2 R_{H}}=\frac{\sqrt{2+z_{e q}}}{1+z_{e q}} \approx 0.018 .
$$

In the original set of variables $\eta$ and $k$, the initial conditions to solve the mode equation are the vacuum conditions to be set at $\eta_{i n i}=-\infty$ :

$$
\begin{gathered}
v_{k}\left(\eta_{i n i}\right)=\frac{e^{-i k \eta_{i n i}}}{\sqrt{2 k}}, \\
\left.\frac{d v_{k}}{d \eta}\right|_{\eta=\eta_{i n i}}=-i \sqrt{\frac{k}{2}} e^{-i k \eta_{i n i}} .
\end{gathered}
$$

Using the new variables $\bar{\eta}$ and $n$, the initial conditions to solve Eq. (38) for one of the two polarization modes are

$$
\begin{aligned}
v_{n}\left(\bar{\eta}_{i n i}\right) & =\sqrt{\frac{R_{H}}{n}} e^{-i n \bar{\eta}_{i n i}}, \\
\left.\frac{d v_{n}}{d \bar{\eta}}\right|_{\bar{\eta}=\bar{\eta}_{i n i}} & =-i \sqrt{n R_{H}} e^{-i n \bar{\eta}_{i n i}} .
\end{aligned}
$$

We have checked that we can, in this case, numerically consider $-\infty$ as any value of $\bar{\eta}_{i n i}<-3$.

The present value of $\bar{\eta}, \bar{\eta}_{0}$, is obtained by solving Eq. (39) for $\bar{\eta}=\bar{\eta}_{0}$, which results in

$$
\bar{\eta}_{0}=-\frac{\sqrt{2-z_{e q}}+2+z_{e q}}{1+z_{e q}} \approx 0.982 .
$$

We can constrain the free parameter $\bar{\eta}_{b}$ by demanding that the end of the bouncing phase happens between the Planck time $\left(z \approx 10^{32}\right.$ and the beginning of nucleosynthesis $\left(z \approx 10^{10}\right)$, which results in

$$
10^{-31} \lesssim \bar{\eta}_{b} \lesssim 10^{-9} .
$$

We have solved Eq. (38) from $\bar{\eta}_{i n i}$ to $\bar{\eta}_{0}$ for four different values of $\bar{\eta}_{b}$ in this range: $10^{-30}, 10^{-24}, 10^{-18}$ and $10^{-12}$.

In terms of the new variables $\bar{\eta}$ and $n$, the expression for the density parameter in Eq. (32), for one of the two possible polarizations, can be rewritten as 


$$
\begin{aligned}
& \Omega_{G W}(n, \bar{\eta})=\frac{\ell_{\mathrm{P} 1}^{2}}{2 \pi^{2} a^{2}} \frac{n^{3}}{32 R_{H}^{5} \mathcal{H}^{2}}\left\{\left|\frac{d v_{n}}{d \bar{\eta}}\right|^{2}-2 R_{H} \mathcal{H} \times\right. \\
&\left.\times\left[v_{n} \frac{d v_{n}^{*}}{d \bar{\eta}}+v_{n}^{*} \frac{d v_{n}}{d \bar{\eta}}\right]+\left(n^{2}+4 R_{H}^{2} \mathcal{H}^{2}\right)\left|v_{n}\right|^{2}\right\}(47)
\end{aligned}
$$

For $\bar{\eta}=\bar{\eta}_{0}$, this expression reduces to

$$
\begin{array}{r}
\Omega_{G W}\left(n, \bar{\eta}_{0}\right)=\frac{l_{p}^{2}}{64 \pi^{2}} n^{3} a_{0} H_{0}^{3}\left\{\left|\frac{d v_{n}}{d \bar{\eta}}\right|^{2}-2\left[v_{n} \frac{d v_{n}^{*}}{d \bar{\eta}}+\right.\right. \\
\left.\left.+v_{n}^{*} \frac{d v_{n}}{d \bar{\eta}}\right]+\left(n^{2}+4\right)\left|v_{n}\right|^{2}\right\}\left.\right|_{\bar{\eta}=\bar{\eta}_{0}}
\end{array}
$$

\section{Analytical Results}

One can anticipate the spectral behavior of the above quantities through some analytical reasonings. Note first that the spectral index of tensor perturbations at the moment they get smaller than the curvature scale in the expanding phase of the above quantum bouncing models was calculated in Ref. [4], and it reads

$$
n_{T}=\frac{12 w}{1+3 w},
$$

where $w$ is the equation of state parameter of the fluid which dominates the cosmic evolution when the mode is getting bigger than the curvature scale of the Universe in the contracting phase. When the fluid is dust, $n_{T}=0$, when it is radiation, $n_{T}=2$.

The power spectrum is

$$
\mathcal{P} \propto n^{3}\left|\mu_{n}\right|^{2} \equiv\left(\Delta h_{n}\right)^{2} \propto n^{n_{T}},
$$

where $\Delta h_{n}$ is the strain of the gravitational wave with wavenumber $n$. Hence, one can obtain the spectra of the strain at the moment $\eta_{c}$ of curvature scale crossing at dust domination and radiation domination from Eq. (49), and they are $\Delta h_{n}\left(\eta_{c}\right) \propto n^{0}$ and $\Delta h_{n}\left(\eta_{c}\right) \propto n$, respectively.

However, we need the strain today, $\Delta h_{n}\left(\eta_{0}\right)$. Note that the curvature scale is given by $l_{c} \equiv\left(a^{3} / a^{\prime \prime}\right)^{1 / 2}$ (which is equal to the Hubble radius in the case of a perfect fluid dominated classical model), and it is comparable to the physical wavelength of the mode $k=n / R_{H}$, $l_{\text {phys }}=a / k$, when $k^{2}=a^{\prime \prime} / a$. Hence, for $l_{\text {phys }}<l_{c}$, or $k^{2}>a^{\prime \prime} / a, v_{k}$ oscillates (see the mode equation (24) for $\mathcal{K}=0)$. Therefore, as $v_{k}=a \mu_{k}$, one has that $\left|\mu_{k}\right|\left(\eta_{0}\right) a\left(\eta_{0}\right)=\left|\mu_{k}\right|\left(\eta_{c}\right) a\left(\eta_{c}\right)$ after curvature scale crossing at the expanding phase. Note that $a\left(\eta_{c}\right)$ as a function of $k$ can be obtained through

$l_{\text {phys }}\left(\eta_{c}\right)=a\left(\eta_{c}\right) / k=l_{c}\left(\eta_{c}\right) \propto R_{H}\left(\eta_{c}\right) \propto t\left(\eta_{c}\right) \propto a^{3 / 2}\left(\eta_{c}\right)$ when $\eta_{c}$ is in the dust dominated era, and

$$
l_{\text {phys }}\left(\eta_{c}\right)=a\left(\eta_{c}\right) / k=l_{c}\left(\eta_{c}\right) \propto R_{H}\left(\eta_{c}\right) \propto t\left(\eta_{c}\right) \propto a^{2}\left(\eta_{c}\right)
$$

when $\eta_{c}$ is in the radiation dominated era. Hence one gets that $a\left(\eta_{c}\right) \propto k^{-2}$ and $a\left(\eta_{c}\right) \propto k^{-1}$ for dust and radiation dominated eras, respectively. Therefore, in terms of the frequency $f=k /\left(2 \pi a_{0}\right)=n H_{0} /(4 \pi)$, the transfer function for the strain,

$$
\Delta h_{f}\left(\eta_{0}\right)=T_{f} \Delta h_{f}\left(\eta_{c}\right),
$$

which is the same as the one for $\left|\mu_{f}\right|$, see Eq. (50), reads

$$
T_{f} \propto\left(\frac{H_{0}}{f}\right)^{2}\left(1+\frac{f}{f_{\mathrm{eq}}}\right),
$$

where $f_{\text {eq }}=H_{\text {eq }} a_{\text {eq }} / a_{0} \approx 10^{-16} \mathrm{~Hz}$ and $H_{0} \approx 10^{-18} \mathrm{~Hz}$.

Finally, the potential $V(\bar{\eta})=\left(d^{2} a / d \bar{\eta}^{2}\right) / a$ of Eq. (38) derived from Eq. (39) reads

$$
V(\bar{\eta})=\frac{2\left[\frac{1}{\bar{\eta}_{*}}+\frac{\bar{\eta}_{b}^{2}}{\left(\bar{\eta}_{b}^{2}+\bar{\eta}^{2}\right)^{3 / 2}}\right]}{\frac{\bar{\eta}^{2}}{\bar{\eta}_{*}}+2 \sqrt{\bar{\eta}_{b}^{2}+\bar{\eta}^{2}}}
$$

and it has a maximum value at $\bar{\eta}=0, V_{\max } \approx \bar{\eta}_{b}^{-2}$. We see therefore that modes with $n>1 / \bar{\eta}_{b}$ do not feel the potential at any time, for they correspond to wavelengths much smaller than the maximum curvature scale of the model. Hence, they are always oscillating with spectrum given by the initial vacuum state,

$$
v_{n}(\eta)=\frac{e^{-i n \bar{\eta}}}{\sqrt{2 n}}
$$

and there is no graviton creation above these frequencies. Therefore, we make a cutoff at the point $n>1 / \bar{\eta}_{b}$, so that there is no zero-point contributions to the energy density, and the vacuum spectrum $\Omega_{G W}$ is clearly finite. Actually, it is important to stress that such zero-point contribution would affect only the high frequency sector of our results.

From Eq. (50), one then infers that the strain $\Delta h_{n}$ has spectrum $n$ for $n>1 / \bar{\eta}_{b}$, independently of $\eta$. As the bounce itself has a duration much smaller then the classical dust-radiation evolution, almost all modes that do not enter the curvature scale after the beginning of classical radiation domination in the expanding phase have the above spectrum.

Noting that our model is symmetric with respect to the bounce, implying that wavelengths which leave the curvature scale before the bounce at some fluid domination era enter the curvature scale after the bounce at the same fluid domination, we can summarize the results concerning the spectrum of the strain in the following way: 
i) $H_{0} \approx 10^{-18} \mathrm{~Hz}<f<10^{-16} \mathrm{~Hz} \approx f_{\text {eq }}$; $\Delta h_{f}\left(\bar{\eta}_{0}\right) \propto f^{-2} f^{0}=f^{-2}$.

ii) $f_{\mathrm{eq}} \approx 10^{-16} \mathrm{~Hz}<f<f_{\max }=H_{0} / \bar{\eta}_{b}$; $\Delta h_{f}\left(\bar{\eta}_{0}\right) \propto f^{-1} f=f^{0}$.

iii) $f_{\max }=H_{0} / \bar{\eta}_{b}<f ; \quad \Delta h_{f}\left(\bar{\eta}_{0}\right) \propto f$.

From the above spectrum, one can easily obtain the spectrum for the energy density parameter of gravitational waves from the relation

$$
\Omega_{G W}\left(f, \bar{\eta}_{0}\right) \propto f^{2}\left[\Delta h_{f}\left(\bar{\eta}_{0}\right)\right]^{2} .
$$

These results are confirmed by the numerical calculation we show in the sequel.

\section{Numerical Results}

Fig. 1 shows the value of the density parameter as a function of frequency $f$ for the four different values of $\bar{\eta}_{b}$ we are considering. Fig. 2 shows the results of the strain amplitude

$$
\left[\Delta h_{n}\left(\bar{\eta}_{0}\right)\right]^{2}=6\left(\frac{a_{0} H_{0}}{n}\right)^{2} \Omega_{G W}\left(n, \bar{\eta}_{0}\right)
$$

Both figures are in accordance with our previous analytical considerations.

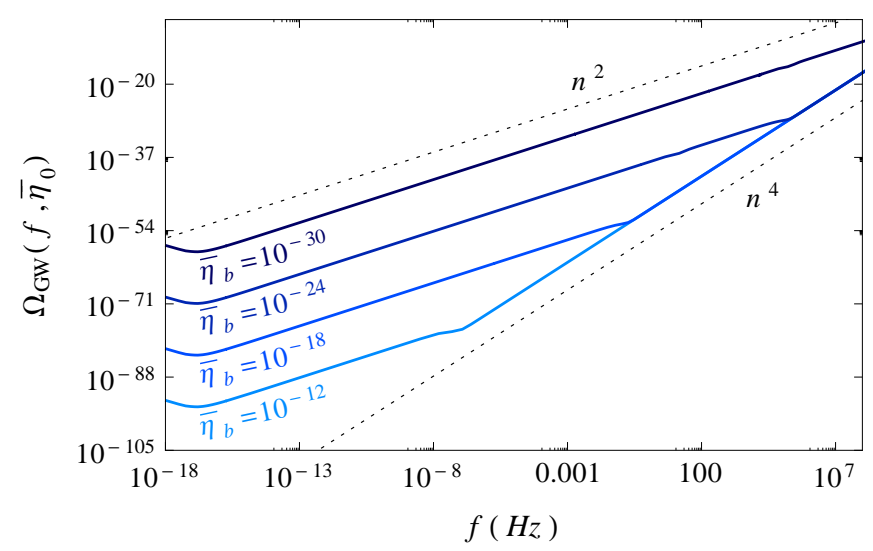

FIG. 1. Energy density parameter as a function of frequency for the primordial gravitational waves produced in our model. Each curve shows the results obtained for the indicated value of $\bar{\eta}_{b}$. The dashed lines show for comparison two power laws proportional to $n^{2}$ and $n^{4}$.

In order to establish the possibility of detecting the primordial gravitational waves produced in our theoretical model, we show in Fig. 3 a zoom of the upper part of Fig. 1, together with other theoretical predictions and experimental sensitivities.

We see that only for the lowest value of $\bar{\eta}_{b}$ we studied, $\bar{\eta}_{b}=10^{-30}$, and for a very high value of frequency, does our result reach the upper limits predicted by the

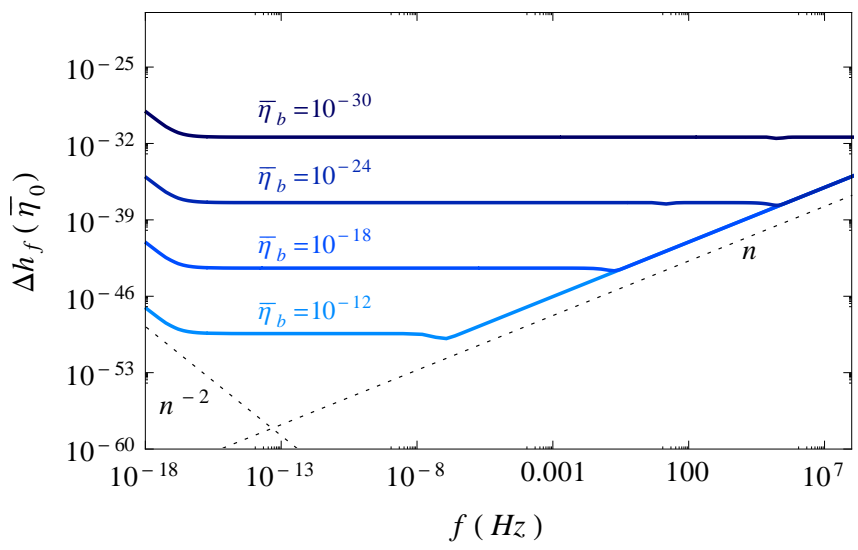

FIG. 2. Strain amplitude as a function of frequency for the same primordial gravitational waves produced in our model. Each curve shows the results obtained for the indicated value of $\bar{\eta}_{b}$. The dashed lines show for comparison two curves proportional to $n^{-2}$ and $n$.

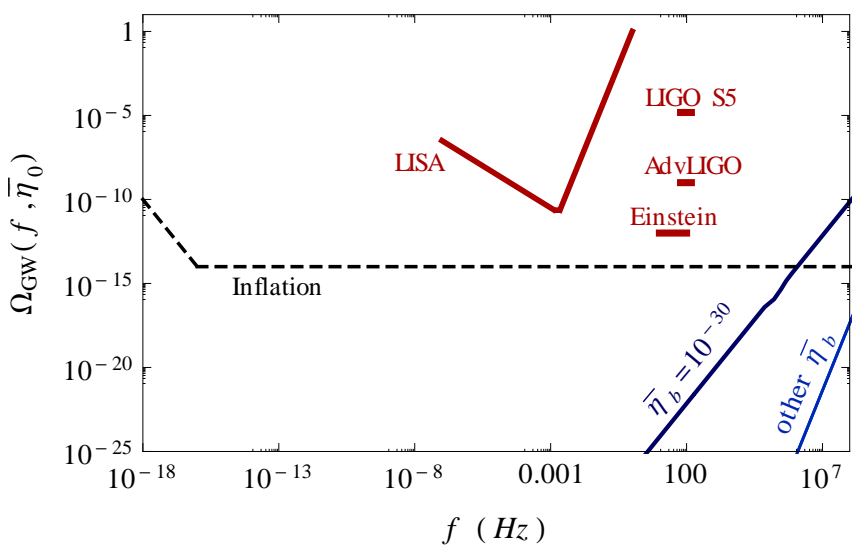

FIG. 3. Comparison of our results (blue curves at the bottom right) with experimental sensitivities (red curves) and a prediction the upper limits on the spectrum of primordial gravitational waves generated in inflationary models (black dashed curve). The red curves show the sensitivities achieved by LIGO's 5th run and the ones predicted for Advanced LIGO, the Einstein Telescope and LISA [21]. See 22] and references therein.

inflationary model. Not even in this extreme case, is the stochastic background strong enough to be directly detected by present and future planned experiments. Nonetheless, we must emphasize that, while our calculations are exact, the predictions presented for inflation are only upper limits and could, therefore, be much lower than the curve presented in Fig. [3. 


\section{CONCLUSIONS}

In this paper we have calculated the amplitude and spectrum of energy density and strain of relic gravitons in a quantum bouncing cosmological model. The strain spectrum of this quantum bouncing model is different from the cyclic and inflationary scenarios. While these two models have spectra $\approx k^{-2}, \approx k^{-1}$ at dust domination, and $\approx k^{-1}, \approx k^{0}$ at radiation domination, respectively, our model have spectra $\approx k^{-2}$ and $\approx k^{0}$ at the same eras.

One possible different scenario in which the power of relic gravitons could be enhanced could be obtained by adding to the matter content of the model some amount of stiff matter, which has primordial spectral index $n_{T}=$ 2. This will be the subject of future investigations.

As a final point, as in the cyclic ekpyrotic model, the resulting amplitude is too small to be detected by any gravitational wave detector. In particular, the sensitivity of the future third generation of gravitational wave detectors, as for example the Einstein Telescope, could reach $\Omega_{\mathrm{GW}} \sim 10^{-12}$ at the frequency range $10-100 \mathrm{~Hz}$ to an observation time of $\sim 5$ years and with a signalto-noise ratio $(\mathrm{S} / \mathrm{N}) \sim 3$. Therefore, any detection of relic gravitons, in this frequency range, will rule out this type of quantum bouncing model as a viable cosmological model of the primordial universe.

\section{ACKNOWLEDGMENTS}

DB thanks the Brazilian agency FAPESP, grant 2009/15612-6 for financial support, and ICRA-CBPF for its kind hospitality. BBS would like to acknowledge the financial support of CAPES under grant number CAPES-PNPD 2940/2011 and to thank CNPq for the 2010-2011 PCI fellowship. NPN would like to thank $\mathrm{CNPq}$ for financial support. ODM would like to thank the Brazilian agency CNPq for partial financial support (grant 300713/2009-6). The authors also thank Sandro Vitenti for his kind help with numerical methods.
[1] M. Novello and S. E. Perez Bergliaffa, Phys. Rep. 463, 127 (2008).

[2] R. Brandenberger and F. Finelli, J. High Energy Phys. 11 (2001) 056; P. Peter and N. Pinto-Neto, Phys. Rev. D 66, 063509 (2002); F. Finelli, JCAP 0310, 011(2003); V. Bozza and G. Veneziano, Phys. Lett. B 625, 177 (2005); V. Bozza and G. Veneziano, JCAP 09, 007 (2005); F. Finelli and R. Brandenberger, Phys. Rev. D 65 (2002) 103522; S. Tsujikawa, R. Brandenberger and F. Finelli, Phys. Rev. D 66, 083513 (2002); P. Peter, N. Pinto-Neto and D. A. Gonzalez, JCAP 0312, 003 (2003); J. Martin, P. Peter, Phys. Rev. Lett 92, 061301 (2004); J Martin, P Peter, N Pinto-Neto and D. J. Schwarz, Phys. Rev. D 65, 123513 (2002); J Martin, P Peter, N Pinto-Neto and D. J. Schwarz, Phys. Rev. D 67, 028301 (2003); C. Cartier, R. Durrer and E. J. Copeland, Phys. Rev. D. 67, 103517 (2003); L. E. Allen and D. Wands, Phys. Rev. D 70, 063515 (2004); T. J. Battefeld, and G. Geshnizjani, Phys. Rev. D 73, 064013 (2006); A Cardoso and D Wands, Phys. Rev. D 77, 123538 (2008); Y Cai, T Qiu, R Brandenberger, Y Piao and X Zhang, J. Cosmol. Astropart. Phys. 03 (2008) 013.

[3] L. A. Boyle, P. J. Steinhardt and N. Turok, Phys. Rev. D 69, 127302 (2004).

[4] P. Peter, E. J. C. Pinho and N. Pinto-Neto, Phys. Rev. D 73, 104017 (2006) arXiv:gr-qc/0605060.

[5] E. J. C. Pinho and N. Pinto-Neto, Phys. Rev. D 76 023506 (2007); P. Peter, E. J. C. Pinho, and N. PintoNeto, Phys. Rev. D 75023516 (2007).

[6] P. Peter, E. Pinho, N. Pinto-Neto, JCAP 0507, 014 (2005). hep-th/0509232.

[7] B. F. Schutz, Jr., Phys. Rev. D2 2762 (1970); Phys. Rev. D4, 3559 (1971).

[8] V. G. Lapchinskii, V. A. Rubakov, Theor. Math. Phys. 33, 1076 (1977); V. Fock, Theory of Space, Time and
Gravitation (Pergamon, London, (1959).

[9] I. M. Khalatnikov, An Introduction to the Theory of Super-fluidity (W. A. Benjamin, New York, 1965).

[10] V. F. Mukhanov, H. A. Feldman, and R. H. Brandenberger, Phys. Rep. 215, 203 (1992).

[11] See e.g. P. Holland, The Quantum Theory of Motion, Cambridge University Press (Cambridge, UK, 1993) and references therein.

[12] F. T. Falciano and N. Pinto-Neto, Phys. Rev. D 79, 023507 (2009) and arXiv:0810.3542 v2 [gr-qc].

[13] J. Acacio de Barros, N. Pinto-Neto, and M. A. SagioroLeal, Phys. Lett. A 241, 229 (1998).

[14] R. Colistete Jr., J. C. Fabris, and N. Pinto-Neto, Phys. Rev. D62, 083507 (2000).

[15] F.G. Alvarenga, J.C. Fabris, N.A. Lemos and G.A. Monerat, Gen.Rel.Grav. 34, 651 (2002).

[16] A. Guth, S. Y. Pi, Phys. Rev. Lett.49, 1110 (1982); A. A. Starobinsky, Phys. Lett. B 117, 175 (1982); S. Hawking, Phys. Lett. B 115, 295 (1982); J. M. Bardeen, P. J. Steinhardt, and M. S. Turner, Phys. Rev. D28, 679 (1983).

[17] V. A. Rubakov, M. V. Sazhin, A. V. Veryaskin, Phys. Lett. B115, 189-192 (1982); R. Fabbri, M. d. Pollock, Phys. Lett. B125, 445-448 (1983); L. F. Abbott, M. B. Wise, Nucl. Phys. B244, 541-548 (1984); B. Allen, Phys. Rev. D37, 2078 (1988); L. P. Grishchuk, Phys. Rev. Lett. 70, 2371-2374 (1993), gr-qc/9304001; M. S. Turner, M. J. White, J. E. Lidsey, Phys. Rev. D48, 4613-4622 (1993), astro-ph/9306029.

[18] M. Giovannini, PMC Phys. A4, 1 (2010). arXiv:0901.3026 [astro-ph.CO]].

[19] M. Maggiore, Phys. Rept. 331, 283-367 (2000). gr-qc/9909001.

[20] V. Mukhanov, "Physical foundations of cosmology," Cambridge University Press (2005).

[21] [LISA is not a project anymore. A new mission called 
European New Gravitational Wave Observatory (NGO) has been derived from the previous LISA proposal. This new mission whose informal name is "eLISA" will survey for the first time the low-frequency gravitational wave band (about $0.1 \mathrm{mHz}$ to $1 \mathrm{~Hz}$ )]; Amaro-Seoane P. et al.,
2012, Class. Quantum Grav., 29, 124016 - eLISA/NGO. [22] The LIGO Scientific Collaboration and The Virgo Collaboration, Nature 460, 990-994 (2009); B. Sathyaprakash et al., "Scientific Objectives of Einstein Telescope", Class. Quantum Grav. 29, 124013, 2012. 